Article

\title{
The Field-Induced Stop-Bands and Lasing Modes in CLC Layers with Deformed Lying Helix
}

\section{Serguei P. Palto}

Shubnikov Institute of Crystallography of Federal Scientific Research Centre "Crystallography and Photonics" of Russian Academy of Sciences, Leninskii pr.59, Moscow 119333, Russia; serguei.palto@gmail.com

Received: 19 August 2019; Accepted: 4 September 2019; Published: 8 September 2019

\begin{abstract}
Waveguide optical properties of a cholesteric liquid crystal (CLC) layer with a deformed lying helix (DLH) have been studied by numerical simulations using the finite difference time domain method. The DLH structure, when the helix's axis is oriented in plane of a CLC layer, is induced by an electric field in a virtual CLC cell with periodic (planar/homeotropic) boundary conditions at one of the alignment surfaces. This in-plane helical structure is stable only in a permanently applied electric field providing the helix deformation. In this work the polarized light reflectance spectra have been studied at different electric fields and light impingement into a waveguide formed by the DLH layer. It is found that for light propagating along the helix axis the reflectance spectrum has multiple stop-bands centred at wavelengths $\lambda_{j}=\frac{2 p\langle n\rangle}{j}$, which is different from set of bands located at $\lambda_{j}=\frac{p\langle n\rangle}{j}$, and characteristic of CLC spectra for the Grandjean-plane textures subjected to distortion by an electric or magnetic field perpendicular to the helix axis, where $j$ is a natural number, $p$ is the helix pitch and $\langle n\rangle$ is the average refractive index. Each of the higher order $(j>1)$ bands consists of three polarization-dependent sub-bands. In the case of an amplifying CLC DLH layer, depending on an extent of the helix deformation, the lasing modes can be excited at different edges of the sub-bands. While at the strongest deformation the lasing is preferable at the edges of the central sub-band; a lower extent of deformation makes favourable conditions for the lasing at edges of the two other sub-bands.
\end{abstract}

Keywords: cholesteric liquid crystals; deformed lying helix; photonic liquid crystals; liquid crystal lasers

\section{Introduction}

Aligned cholesteric liquid crystals (CLCs) are well-known representatives of one-dimensional photonic crystals. Their photonic properties are due to both the optical anisotropy and helical structure of the director field, which result in an appearance of a spectral band gap (stop-band), within which the light propagation along the axis of the cholesteric helix is forbidden for one of the circular polarizations [1]. At the edges of the stop-band, the group velocity of the light is strongly decreased. The last leads to an enhanced density of states and many photonic effects, such as low-threshold lasing [2-10].

Typically, at the homogeneous boundary conditions, CLCs form planarly aligned layers with Grandjean-plane texture, in which the helix axis is oriented along the normal to the CLC layer. However, for many electrooptical and photonic applications the CLC layers with the in-plane helix axis are desirable. Such CLC layers are known as ULH (uniform lying helix) layers [11]. The ULH layers are quite interesting for the low-threshold lasing because of more efficient transverse optical pumping [5].

During rather long periods of time, different approaches were proposed in order to get the ULH layers [11-15]. One of serious problems in getting the ULH is associated with its thermodynamic instability [13]. Typically, additional electric fields and temperature treatment are necessary in order 
to get the ULH textures. Recently, it was demonstrated that CLC layers with the helix axis along the normal plane can be transformed to the layers with the in-plane helix axis under a permanently applied electric field in cells with the periodic (planar/homeotropic) boundary conditions [16]. However, because the electric field is permanently applied, the lying helix is deformed (it is why such CLC layers were called in [16], deformed lying helix (DLH) layers).

This paper is dedicated to the study of optical properties of the DLH layers by numerical simulations. In particular, the spectral structure of stop-bands and lasing modes which can be excited at different magnitudes of the applied electric field for light propagating in a waveguide regime are studied. It is worth it to mention that in $[17,18]$, it was theoretically shown that in case of a magnetic or electric field applied perpendicular to the helix's axis the optical spectrum is characterized by the appearance of higher order stop-bands. Moreover, each of the stop-bands consists of three sub-bands characterized by different sensitivity to the light's polarization state. In reference [19] using a very strong pulsing electric field the appearance of intensive, multiple bands of a higher order, and their triple structure, were confirmed experimentally. Nevertheless, the field-induced bands' composition and their spectral structure remain unknown in case of the DLH layers due to both the waveguiding regime and specific features of the director field deformation.

\section{Numerical Simulation Methods}

The simulations include two stages. At the first stage a DLH transition [16] is simulated, when the virtual CLC layer with the planar texture (helix axis along the layer normal) is transformed by an electric field to the DLH state. At the second stage the optical and lasing properties of the DLH state are studied for different extents of the helix deformation.

The numerical simulations are made using the author's LCDTDK software, which has had a rather long developing and testing curve (of about 20 years). The software allows simulations for both texture (liquid crystal (LC) director distribution) and optical problems for LC systems of complicated designs.

The parameters of the virtual CLC in this study correspond to a real CLC material that was designed and experimentally studied in the work [16]. This experimental CLC was made on the basis of the popular E7 (Merck) nematic mixture by doping it with both optically active compound, providing a natural helix pitch of $260 \mathrm{~nm}$, and DCM lasing dye. Thus, all the parameters (elastic coefficients, low frequency dielectric anisotropy and optical anisotropy) used in the current simulations are close to values characteristic of the E7 LC. The boundary conditions are also close to those achieved experimentally in [16]. Despite some preliminary experimental study of the lasing in the DLH systems done in [16], the results of this numerical study are still to be tested experimentally.

\subsection{Simulations of the DLH Transition}

The texture calculations are based on solving general equations of the Elastic Liquid Crystal Continuum Theory for 3D inhomogeneous LC layers at arbitrarily defined boundary conditions (topography of the alignments' surfaces, profiles of spatial distribution of the easy axes and anchoring strength, and multiple electrodes-systems can be defined). Some details of the approaches used for a simplified one-dimensional problem were described in [20]. Different optical problems can be solved using the $4 \times 4$ matrix Berreman method [21] implemented in the software. However, because the Berreman approach has limitations in the cases of 2D and 3D inhomogeneous LC systems, the finite difference time domain (FDTD) method is implemented as well [22]. Namely the FDTD method is used in the current simulations, so some important aspects of the method are described below in detail.

It was not the goal to describe all features of the software, so herein only some principal points important for understanding simulated results are mentioned. 
The governing equations for finding the LC director $\left(\mathbf{n}=\left(n_{x}, n_{y}, n_{z}\right)\right)$ distribution are as follows:

$$
\begin{aligned}
& -\frac{\partial(F+g)}{\partial n_{i}}+\frac{d}{d x}\left(\frac{\partial(F+g)}{\partial n_{i} / \partial x}\right)+\frac{d}{d y}\left(\frac{\partial(F+g)}{\partial n_{i} / \partial y}\right)+\frac{d}{d z}\left(\frac{\partial(F+g)}{\partial n_{i} / \partial z}\right)=\gamma \frac{d n_{i}}{d t} \\
& i \in\{x, y, z\}, g \equiv \frac{1}{2} \mu\left(1-\sum_{i} n_{i}^{2}\right)=0
\end{aligned}
$$

where $\mu$ is Lagrange multiplier that is due to the constraint

$$
n_{x}^{2}+n_{y}^{2}+n_{z}^{2}=1
$$

$\gamma$ is the rotational viscosity of $\mathrm{LC}$, and $F$ is the free energy density, expressed as:

$$
\begin{aligned}
& F=\frac{1}{2}\left(K_{1}(\nabla \cdot \mathbf{n})^{2}+K_{2}\left(\mathbf{n} \cdot(\nabla \times \mathbf{n})+q_{0}\right)^{2}+K_{3}(\mathbf{n} \times(\nabla \times \mathbf{n}))^{2}-(\varepsilon \mathbf{E}) \cdot \mathbf{E}\right) \\
& -\mathbf{E}\left(e_{1}(\nabla \cdot \mathbf{n}) \mathbf{n}-e_{3}(\mathbf{n} \times(\nabla \times \mathbf{n}))\right)
\end{aligned}
$$

where $K_{1}, K_{2}$ and $K_{3}$ are splay, twist and bend elastic coefficients, respectively; $\varepsilon$ is the dielectric tensor, $q_{0}=2 \pi / p_{0}$ is a wavenumber associated with the natural pitch $p_{0}$ of the cholesteric helix; $e_{1,3}$ are the flexoelectric coefficients; and $\mathbf{E}$ is the electric field vector. The elastic parameters used in this work are close to ones for the well known E7 LC material: $K_{1}=11 \mathrm{pN}, K_{2}=7 \mathrm{pN}$ and $K_{3}=17 \mathrm{pN}$. The flexoelectricity was neglected in this work for reasons discussed in [16], so it was assumed that $e_{1}=e_{3}=0$.

In this work the rigid boundary conditions (infinite anchoring strength) were used, so the set of equations to solve the boundary problem is skipped in this article. However, to get the DLH transition, the periodic boundary conditions, when the easy axis is flipped between the planar and homeotropic state, are necessary. The periodic conditions used are shown in Figure 1 at the $S_{1}$-surface. In practice such periodic conditions can be created by recording the anchoring grating of a period $\Lambda$ using the focused ion beam treatment of a polyimide alignment layer [16]. The wavevector of the anchoring grating providing the periodic boundary conditions is along the $x$-axis of the laboratory $x y z$-frame, while the $z$-axis is perpendicular to the CLC layer. At the second alignment surface $\left(\mathrm{S}_{2}\right)$ the homogeneous homeotropic alignment is used.

As discussed in [16], for the DLH transition to appear it is also important to have the CLC layer thickness $(d)$ comparable with the period of the easy axis flipping $(\Lambda)$. In this work the thickness of the CLC layer and the anchoring grating period are taken to be 750 and $550 \mathrm{~nm}$, respectively. The other important feature of the DLH transition is that the natural pitch $\left(p_{0}\right)$ of the CLC material has to be at least twice lower than $\Lambda$. In this work $p_{0}=260 \mathrm{~nm}$.

Because the director field is not homogeneous, the electric field distribution is also not the homogeneous one. In order to find the electric field distribution, Equations (1) are coupled with the Maxwell equations $\operatorname{rot}(\mathrm{E})=0$ and $\operatorname{div}(\varepsilon \mathrm{E})=0$. Thus, we assume that the $\mathrm{LC}$ is the ideal dielectric with zero free charge density in the bulk.

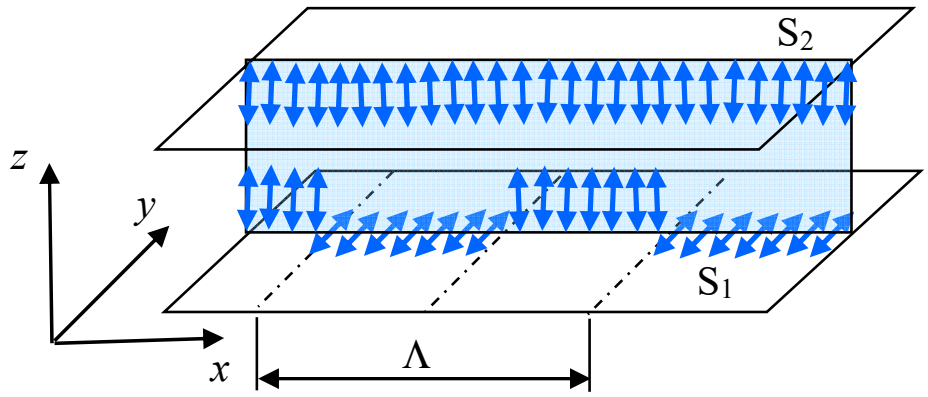

Figure 1. The scheme of the boundary conditions at cholesteric liquid crystal (CLC) layer alignment surfaces $S_{1}$ and $S_{2}$. The distributions of the easy axes are shown by arrows. Infinite anchoring energy (rigid boundary conditions) is used at both surfaces. 
The transition to the DLH state appears when the voltage across the initial CLC layer (the voltage $U$ is applied between virtual electrodes at $S_{1}$ and $S_{2}$ alignment surfaces) is gradually increased (scan-up half-cycle) from zero to some value that is quite close to the helix unwinding voltage, which can be estimated as [23]:

$$
U_{c}=d \frac{\pi^{2}}{p_{0}} \sqrt{\frac{K_{2}}{\varepsilon_{0} \Delta \varepsilon}}
$$

where $\varepsilon_{0} \cong 8.85 \times 10^{-12} \mathrm{~F} / \mathrm{m}$ is the dielectric permittivity of the free space and $\Delta \varepsilon=\varepsilon_{\|}-\varepsilon_{\perp}$ is the low frequency dielectric anisotropy of the CLC material $\left(\varepsilon_{\|}\right.$and $\varepsilon_{\perp}$ are principal dielectric components of the tensor at low frequency). For our virtual CLC layer, $U_{c} \cong 7 \mathrm{~V}$.

The DLH state remains in some interval of the applied voltages, when after the scan-up half-cycle, the voltage is gradually decreased (scan-down half-cycle) [16]. It is also worth mentioning that the vector model does not allow transitions between the topologically nonequivalent states, so in the simulations the helix can remain at voltages even higher than $U_{c}$. In our case the studied interval of the voltages was between 7 and $4 \mathrm{~V}$. In that voltage interval the extent of the helix deformation is strongly changed, so one can see how the optical properties are influenced by quite different degree of the deformation.

\subsection{FDTD Simulations}

The FDTD method is widely used in modern optical simulations. It is based on a direct solution of the Maxwell equations in the time domain. Currently the basics of the FDTD method are described in numerous sources. I would like to recommend the EM lab site [22], where one can find quite detailed and useful lectures explaining different aspects of the method and possible numerical implementations, which can be a good starting point for developing complicated software.

The author's FDTD software module used in this work is directly bounded to the texture module briefly described above. The dielectric properties of materials are defined in terms of a spatial distribution of the dielectric tensor components and take into consideration their spectral dispersion. The spectral dispersion is taken in frame of the popular, multiple-oscillator Lorentz-Drude model. In terms of the Lorentz-Drude model the frequency dependence of the principal dielectric tensor components of a liquid crystal are described as:

$$
\varepsilon_{\|, \perp}(\omega)=\varepsilon_{\|, \perp, \infty}+\sum_{m=1}^{N} \frac{A_{m, \|, \perp}}{\omega_{m}^{2}-\omega^{2}+i \omega \Gamma_{m}},
$$

where $\varepsilon_{\|, \infty}$ and $\varepsilon_{\perp, \infty}$ are principal components of the dielectric tensor at the infinite frequency (in case of the spectral dispersion, it is neglected only when these values are used), which correspond to the directions along (subscript " $\|$ ") and perpendicular (subscript " $\perp$ ") to the director. The dispersion is described by contributions from $N$ oscillators, where the $m$-th oscillator is characterized by its frequency $\omega_{m}$, damping coefficient $\Gamma_{m}$ and magnitude $A_{m}$, proportional to the strength of the oscillator. Each of the oscillators gives a contribution to the total polarization $(P(\omega)=\varepsilon(\omega) E(\omega))$ for which there are corresponding time domain equations [22].

$$
\frac{\partial^{2} P_{m, \|, \perp}(t)}{\partial t^{2}}+\frac{\partial}{\partial t}\left(\Gamma_{m} P_{m, \|, \perp}(t)\right)+\omega_{m}^{2} P_{m, \|, \perp}(t)-A_{m, \|, \perp} E_{\|, \perp}(t)=0
$$

Thus, the dependencies described by Equation (5) are transformed to the time domain using the time-dependent polarization field within Maxwell's equations.

Equation (5) is also connected to spectra of the principal absorption coefficients $k_{\|, \perp}(\lambda)$ due to the known relationship:

$$
\varepsilon_{\|, \perp}(\lambda)=\left(n_{\|, \perp}(\lambda)+i \frac{\lambda k_{\|, \perp}(\lambda)}{4 \pi}\right)^{2}
$$


where $n_{\|}$and $n_{\perp}$ are the principal refractive indices for light linearly polarized along and perpendicular to the LC director, respectively; $\lambda$ is the free space light wavelength. The simulation results of this work are presented on a wavelength scale, which, to my mind, is more convenient for further comparison with experimental study of the simulated system.

In current work, in order to simulate the lasing effect, the negative $k(\lambda)$ values corresponding to an amplifying CLC medium are used. In frame of the Lorentz-Drude model, to define the negative $k$ one needs to use negative values of $A_{m}$, which result in the negative imaginary part of the dielectric permittivity tensor components (Figure 2).



Figure 2. Spectral dependencies of the principal components of the complex permittivity tensor used for simulation of the lasing effect.

It is important to say that in current work the spectral dispersion is taken into account only to simulate the lasing effect. In all the other cases the dispersion is neglected in order to simplify the interpretation of the simulated results. Thus, in cases of simulations of the non-amplifying DLH layers, the virtual CLC material is described by frequency-independent values of the principal components of the dielectric tensor $\left(\varepsilon_{\|}=\varepsilon_{\|, \infty}=3.06, \varepsilon_{\perp}=\varepsilon_{\perp, \infty}=2.31\right)$. The last values of the dielectric tensor components correspond to the principal refractive indices $\mathrm{n}_{\|}=1.75$ and $\mathrm{n}_{\perp}=1.52$, which are close to the E7-LC values measured in middle of the visible spectral range (wavelength $\sim 550 \mathrm{~nm}$ ) at room temperature.

The scheme of the FDTD simulated space domain is shown in Figure 3. Because the CLC DLH-layer is homogeneous along the $y$-axis, all the $d / d y$ derivatives vanish, and the optical problem is reduced to the $2 \mathrm{D}$ inhomogeneous problem in the $x z$-plane, which allows for significant increases in efficiency of the FDTD simulations. The CLC DLH-layer (1) of a thickness of $0.75 \mu \mathrm{m}$ and of a length $L=10 \mu \mathrm{m}$ is placed in a medium (2) with a refractive index of 1.46. Because all the principal refractive indices of the CLC are higher, the last one allows for a waveguiding regime for the light impinged into the CLC layer from the unidirectional light source (3). The virtual sensor (4) is placed in the shadow of the light source (3) and registers only the light reflected by the DLH layer. The electrodes used to apply the voltage are neglected in the sense that their refractive indices are set to be the same as for the medium (2). The uniaxial perfectly matched layers (5) prevent reflections from boundaries of the calculated space domain [22]. 


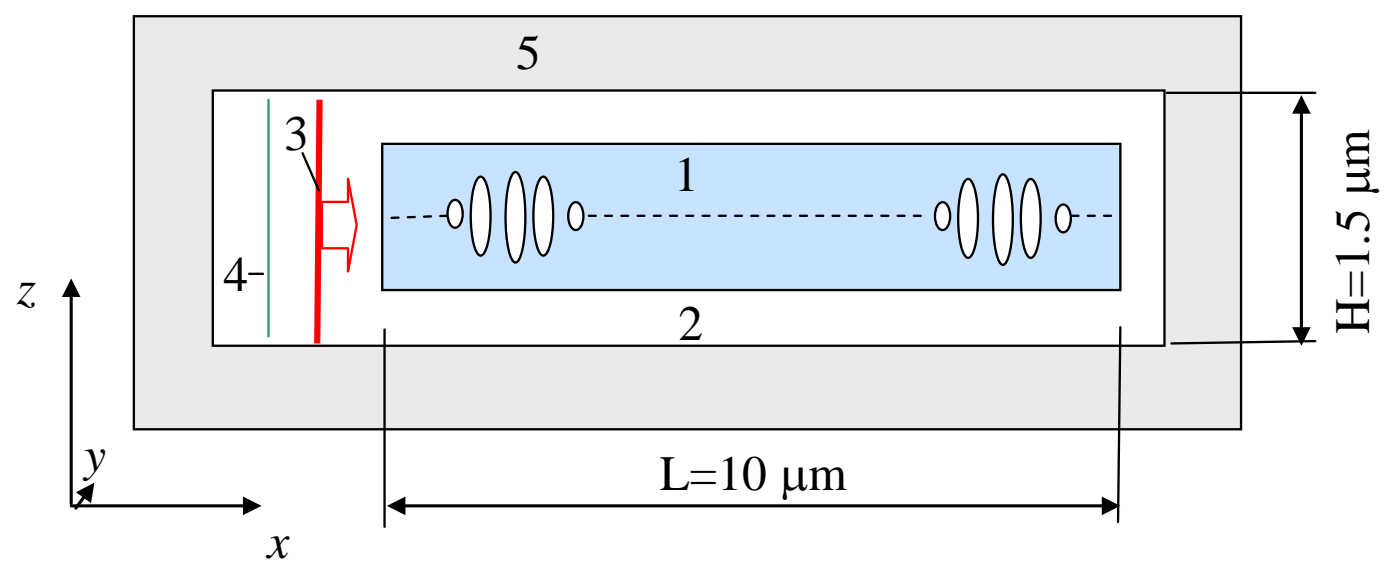

Figure 3. Scheme of the finite time difference domain (FDTD)-simulated domain. 1-CLC deformed lying helix (DLH)-layer; 2-the medium with a refractive index of 1.46; 3-unidirectional light source; 4 -sensor of the reflected light; 5-the uniaxial perfectly matched layers.

The light source (4) generates a pulse of the electromagnetic field propagating along the $\mathrm{x}$-axis. The magnitude of the pulse is constant in the $z$-direction. The pulse represents a sine wave $(\lambda=550 \mathrm{~nm})$ modulated by the Gaussian waveform with 1/e-height duration, $\sim 5 \mathrm{fs}$. The last results in a rather wide spectrum of the generated light, which allows for calculations of the reflectance spectra in a wavelength range of 400 to $2000 \mathrm{~nm}$. The sensor (4) registers across time, the components of the electromagnetic field. To get the reflectance spectra, the ratio of the energy flux in the $x$-direction $\left(P_{x}\right)$ of the electromagnetic field at the sensor $(4)$ to the energy flux irradiated by light source $\left(\mathrm{P}_{\mathrm{ls}}\right)$ is calculated. The values $\mathrm{P}_{\mathrm{x}}$ and $\mathrm{P}_{\mathrm{ls}}$ are calculated versus the wavelength by taking the Fourier transform of the field registered by sensor (4) and finding $x$-components of the Poynting vector at the sensor (4) and light source (3) positions. Because the $x$-components of the Poynting vector for the reflected light are negative, the reflectance magnitudes in spectra shown below are also negative. The spectral resolution in the calculated spectra is defined by total time of registration the electromagnetic field. In our calculations the total registration times are about 1000 and $2000 \mathrm{fs}$ for the reflectance spectra and lasing effect respectively, which correspond to the resolutions of about $1 \mathrm{~nm}$ and $0.5 \mathrm{~nm}$ at a wavelength of $550 \mathrm{~nm}$.

\section{Results and Discussion}

The simulated DLH transition is illustrated in Figure 4. The field-off $(U=0 \mathrm{~V})$ initial distribution of the director is shown in Figure 4a. In this case, the director field is close to that characteristic of the Granjean-plane texture with the helix axis along the layer's normal plane. The difference from the Granjean-plane texture is only near the alignment surfaces, which is caused by the periodic boundary conditions used at the $S_{1}$ surface and the homeotropic alignment at the surface $S_{2}$ (Figure 1). The DLH state shown in Figure $4 \mathrm{~b}$ has been achieved as follows. First the voltage was gradually increased from zero to $9 \mathrm{~V}$ on the simulated time scale of $100 \mathrm{~ms}$. The distribution shown in Figure 4a remains almost unchangeable up to $4 \mathrm{~V}$. Above the voltage $4 \mathrm{~V}$ the undulated texture [16] appeared, and then, at $5 \mathrm{~V}$, it transformed to the DLH state. The DLH state with a different level of helix deformation remains during further increase of the voltage up to $9 \mathrm{~V}$. The DLH still exists during the voltage scan-down half-cycle up to a voltage of $4 \mathrm{~V}$, as shown in Figure $4 \mathrm{~b}$. At a voltage of $7 \mathrm{~V}$ the helical distribution is strongly deformed. In the middle of the layer the $x$-distribution of the director components $n_{z}$ and $n_{y}$ (Figure 5a) are far from the cosine(sine)-waves characteristic of the non-disturbed helix.

From the Fourier transform amplitude spectra shown in Figure $5 b$, one can see that the deformed helical distribution is characterized by a set of rather intensive harmonics located at spatial frequencies $\mathrm{q}_{\Lambda}, 2 \mathrm{q}_{\Lambda}$ and $3 \mathrm{q}_{\Lambda}$, where $\mathrm{q}_{\Lambda}=1 / \Lambda$. 


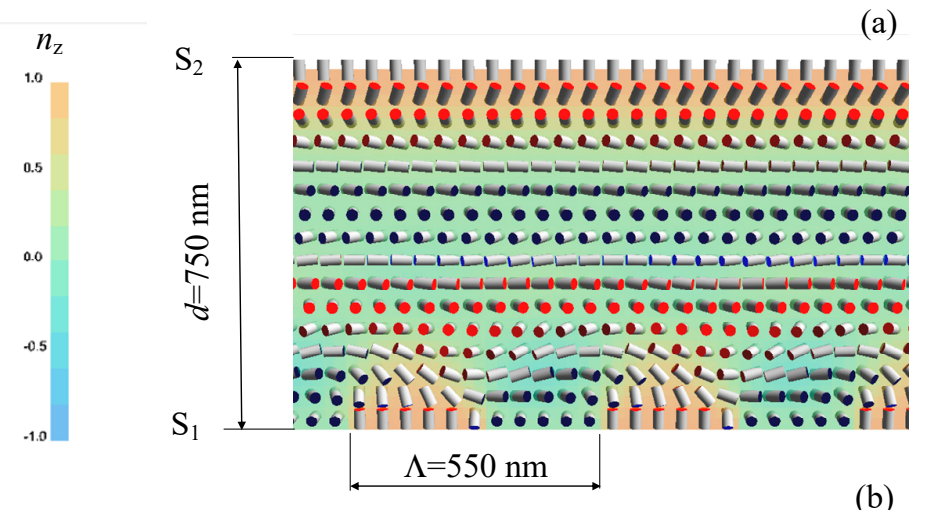

(a)

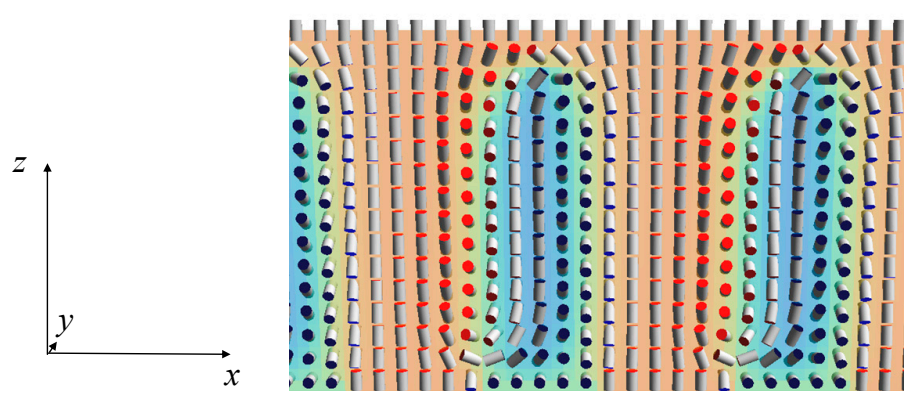

Figure 4. Initial at $U=0 \mathrm{~V}(\mathbf{a})$ and DLH at $U=4 \mathrm{~V}(\mathbf{b})$ (during the scan-down the voltage half-cycle) director field distributions in a CLC layer (thickness $d=750 \mathrm{~nm}$ ). The $S_{1}$ alignment surface provides the periodic (planar/homeotropic) anchoring conditions with the spatial period $\Lambda=550 \mathrm{~nm}$. The $S_{1}$ and $S_{2}$ alignment surfaces provide the infinite anchoring energy. The color scale is given for the $n_{\mathrm{z}}$ component of the LC director. The parameters of the CLC material used in the simulations are as follows: natural pitch $p_{0}=260 \mathrm{~nm}$; elastic constants $K_{1}=11 \mathrm{pN}, K_{2}=8 \mathrm{pN}$ and $K_{3}=17 \mathrm{pN}$; low frequency principal dielectric constants $\varepsilon_{\perp}=6, \varepsilon_{\|}=20$.

(a)
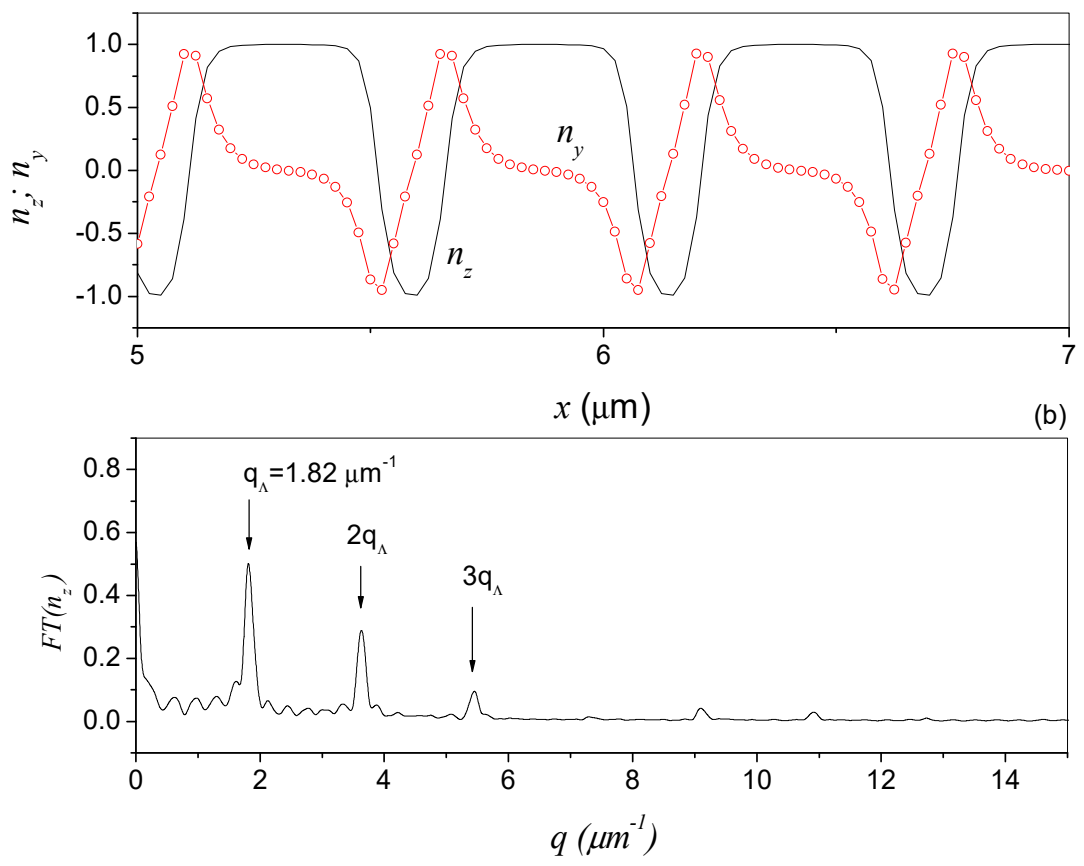

Figure 5. (a) The director distribution along the $x$-direction (due to the periodicity the director distribution is shown only for the central part of the virtual CLC cell from $x=5$ to $x=7 \mu \mathrm{m}$ ) in middle of the CLC DLH-layer at $U=7 \mathrm{~V}$; (b) Fourier transform spectrum characteristic of the spatial distribution of the $z$-component $\left(n_{z}\right)$ of the director along the $x$-axis in the middle of the DLH layer at $U=7 \mathrm{~V}$. 
It is worth mentioning that in case of the Granjean-plane texture, the helix distribution distorted by the electric field perpendicular to the helix axis, is characterized only by the odd harmonics [19], which, as will be shown below, makes a principal difference between sets of the field-induced stop-bands in case of the DLH and the distorted Granjean-plane texture.

Figure 6 shows the reflectance spectra calculated for different strengths of the applied electric field, and as a result, for different extents of helix deformation. The reflectance is sensitive to the polarization state of the incident light. The curves labelled 1 and 2 are for the $z$ - and $y$-linearly polarized light impinged into the CLC layer ( $z$ - and $y$-polarization are equivalent to the transverse magnetic (TM) and transverse electric (TE) polarization, respectively). The spectra show multiple reflection bands, and the first step is to compare the positions of centres of these bands with those for the Granjean-plane texture distorted by the field perpendicular to the helix axis [17]:

$$
\lambda_{j}=p \frac{n_{\|}+n_{\perp}}{2 j} \equiv \frac{p\langle n\rangle}{j}
$$

where $j$ belongs to the set of the natural numbers, $\langle n\rangle=\left(n_{\|}+n_{\perp}\right) / 2$ denotes the average refractive index and $p$ is the helix pitch. In our case of the DLH transition, the helix pitch is equal to the period of the anchoring modulation $\left(p=\Lambda=550 \mathrm{~nm}\right.$ ). Taking the values $n_{\|}=1.75$ and $n_{\perp}=1.52$, one can find from Equation (8) the values $\lambda_{1} \cong 900 \mathrm{~nm}$ and $\lambda_{2}=450 \mathrm{~nm}$. These two bands indeed exist in the spectra shown in Figure 6. However, the number of the existing bands in case of the DLH is larger than that predicted by Equation (8).

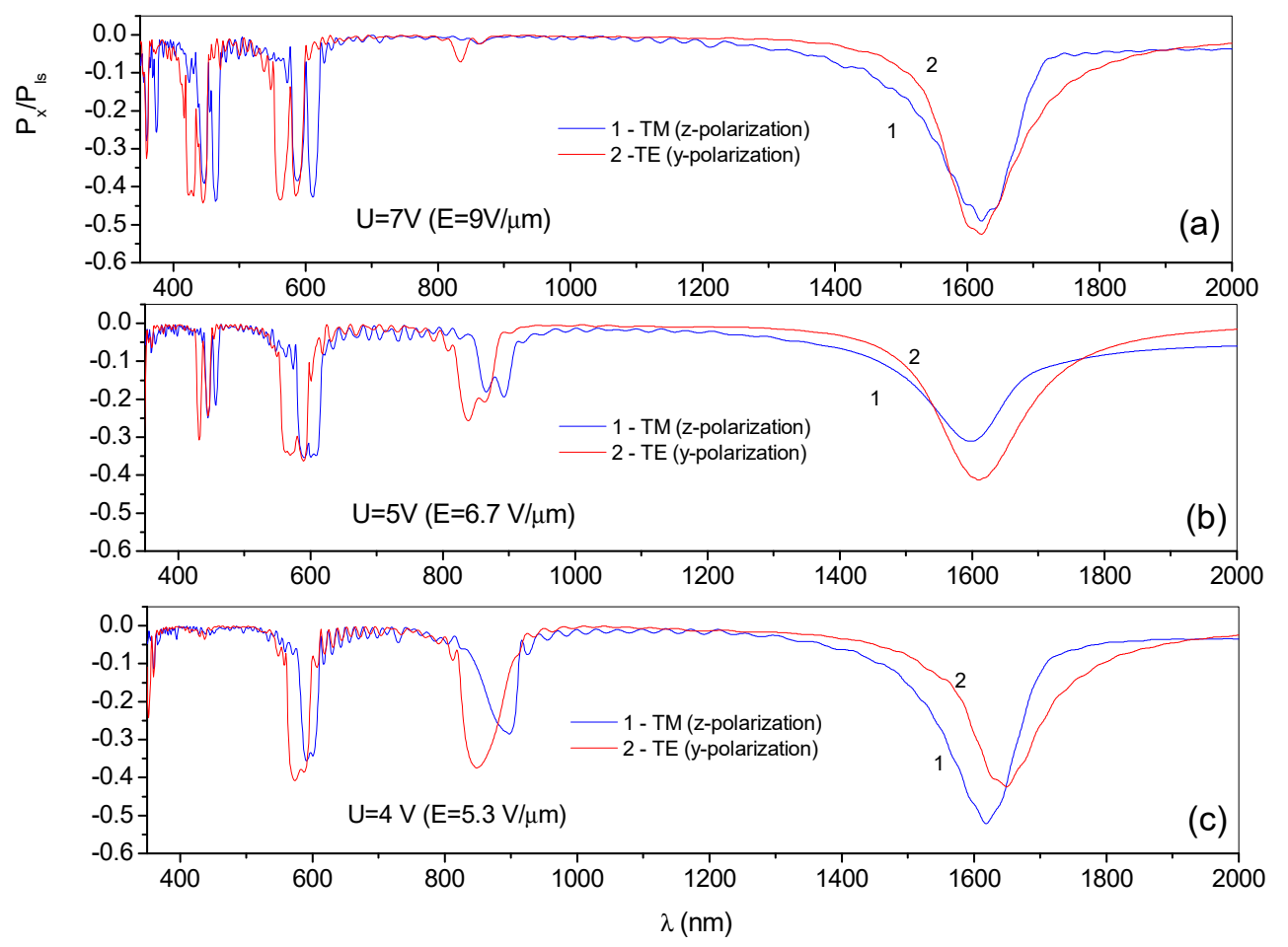

Figure 6. Spectra of the reflected light (the ratio of the reflected light flux $\left(\mathrm{P}_{\mathrm{x}}\right)$ to the magnitude of the flux from light source $\left(\mathrm{P}_{1 \mathrm{~s}}\right)$, calculated using the $x$-components of the Poynting vectors at the lines of the sensor and light source, Figure 3), at different magnitudes of voltage $U$ applied to the CLC DLH layer: (a) $7 \mathrm{~V}$, (b) $5 \mathrm{~V}$ and (c) $4 \mathrm{~V}$. Curves labelled 1 and 2 are for TM- and TE-polarized incident light, respectively.

For example, the two bands centred at wavelengths $\sim 600 \mathrm{~nm}$ and $\sim 1600 \mathrm{~nm}$ cannot be explained by the Equation (8). The origin of these bands can be understood in terms of the light scattering amplitude approach used by de Gennes and Prost [23] on account of the second Fourier harmonic 
$\left(2 q_{\Lambda}\right)$ in the director field of the DLH, shown in Figure $5 b$. The scattering amplitudes are proportional to component magnitudes in Fourier spectrum $\varepsilon(q)$, where $q=k_{i}-k_{s}$ and $k_{i}, k_{s}$ are wavevectors of the incident and scattering waves, respectively. The spatial distributions of the LC dielectric tensor components expressed in terms of the director components are as follows:

$$
\varepsilon_{i j}(x)=\varepsilon_{\perp} \delta_{i j}+\left(\varepsilon_{\|}-\varepsilon_{\perp}\right) n_{i}(x) n_{j}(x)
$$

where $i, j \in\{x, y, z\}, \delta_{i j}=1$ at $i=j$ and $\delta_{i j}=0$ if $i \neq j$. For example, for $\varepsilon_{\mathrm{zz}}(\mathrm{x})$ on account of the first and second harmonics in the spatial distribution of the director field $n_{z}(x)$ :

$$
\varepsilon_{z z}(x)=\varepsilon_{\perp}+\left(\varepsilon_{\|}-\varepsilon_{\perp}\right)\left(C_{1} \cos \left(\frac{2 \pi}{\Lambda} x\right)+C_{2} \cos \left(\frac{4 \pi}{\Lambda} x\right)\right)^{2},
$$

where $C_{1}$ and $C_{2}$ are amplitudes of the first and second harmonics in the spatial distribution of the $z$-component of the director $\left(n_{z}\right)$.

Taking the Fourier transform of $\varepsilon_{z z}(\mathrm{x})$ we get:

$$
\begin{aligned}
& \varepsilon_{z z}(q)=\int_{x} \varepsilon_{z z}(x) e^{-i q x} d x \\
& =\varepsilon_{a} \frac{C_{1} C_{2}}{2} \int_{x}\left(e^{i\left(q+\frac{2 \pi}{\Lambda}\right) x}+e^{i\left(q-\frac{2 \pi}{\Lambda}\right) x}\right) d x+\varepsilon_{a} \frac{C_{1}^{2}}{4} \int_{x}\left(e^{i\left(q+\frac{4 \pi}{\Lambda}\right) x}+e^{i\left(q-\frac{4 \pi}{\Lambda}\right) x}\right) d x \\
& +\varepsilon_{a} \frac{C_{1} C_{2}}{2} \int_{x}\left(e^{i\left(q+\frac{6 \pi}{\Lambda}\right) x}+e^{i\left(q-\frac{6 \pi}{\Lambda}\right) x}\right) d x+\ldots
\end{aligned}
$$

where $\varepsilon_{\mathrm{a}}=\left(\varepsilon_{\|}-\varepsilon_{\perp}\right)$.

It is evident that the integrals at the right side of Equation (11) are not equal to zero in case of

$$
q=\left\{ \pm \frac{2 \pi}{\Lambda}, \pm \frac{4 \pi}{\Lambda}, \pm \frac{6 \pi}{\Lambda} \ldots\right\}
$$

which allows for finding the spectral positions of the reflection bands. Taking into account that for the light propagating along the helix axis, the wavevector of a scattered (reflected) wave is just opposite to the wavevector of an incident wave $\left(k_{i}=-k_{\mathrm{s}}\right)$, we have $q=k_{\mathrm{i}}-k_{\mathrm{s}}=2 k_{i}=4 \pi\langle n\rangle / \lambda$, and on account of Equation (12) one can discern that the set of the reflection bands is centered at wavelengths:

$$
\lambda_{j}=\frac{2\langle n\rangle \Lambda}{j} \equiv \frac{\left(n_{\|}+n_{\perp}\right) p}{j},
$$

where $j$ belongs to set of the natural numbers, and we have taken into account that the helix pitch $p$ is equal to $\Lambda$, and the number of harmonics in the Fourier spectrum of DLH director distribution is not limited just by two harmonics, so $j$ can be higher than three.

The Equation (13) explains the set of the bands shown in Figure 6 for the DLH. It is important to underline, that the case of the director field is represented only by odd spatial harmonics; as for the distorted Granjean-plane texture [19], the scattering amplitude approach leads to a quite different set of bands described by Equation (8). Moreover, looking at the first and third integrals at the right side of the Equation (11) one can see that their values are proportional to the product $C_{1} C_{2}$, which means that the bands with $j=1$ and $j=3$ in Equation (13) are due to the coupling between the first and second harmonics of the DLH director distribution. Namely, this coupling is responsible for the non-zero scattering amplitudes and the bands centred at about 600 and $1700 \mathrm{~nm}$ in the calculated spectra. It is worth mentioning here that the Equation (13) holds for the wave propagating exactly along the helix axis. In case of the waveguiding regime some modes can propagate at a small angle with respect to the helix axis, so the corresponding bands will be shifted to the shorter wavelengths compared to that given by Equation (13). For example, according to Equation (13) at $j=1, n_{\|}=1.75$ 
and $n_{\perp}=1.52, p=550 \mathrm{~nm}$, the $\lambda_{1}=1798 \mathrm{~nm}$, while the first band in the calculated spectra (Figure 6) is centred at a shorter wavelength of about $1600 \mathrm{~nm}$.

It is well visible from the spectra in Figure 6 that each of the bands with $j>1$ has a fine structure. Actually, if we take an arbitrary polarized light, the spectral structure of each band with $j>1$ is represented by three sub-bands. As an example, let us consider in more detail the structure of the band with $j=3$ that is in a range of 550 to $625 \mathrm{~nm}$. In the case that the incident light is z-polarized (TM-polarization), the light is reflected in two sub-bands with the maxima at 610 and $586 \mathrm{~nm}$. The polarizations of light reflected in these two bands are mutually orthogonal, as one can see in Figure $7 \mathrm{~b}$, where the reflectance spectra are shown for the $y$ - and $z$-polarized components of the Poynting vector (curves 1 and 2, respectively). The sub-band at $610 \mathrm{~nm}$ (designated as $\mathrm{B}_{\mathrm{TM}-\mathrm{TM}}$ in Figure $7 \mathrm{~b}$; the subscript "TM-TM" means that the band "B" corresponds to the TM-polarized reflected light in the case of the impinged TM-polarized light) results in reflection of the TM-polarized light, while the band at $586 \mathrm{~nm}$ (designated as $\mathrm{B}_{\mathrm{TM}-\mathrm{TE}}$ ) provides TE-polarized reflected light. If the incident light is $y$-polarized (TE polarization), then the spectrum (Figure 7c) is shifted to the shorter wavelengths, and we again have two sub-bands designated as $B_{\mathrm{TE}-\mathrm{TE}}$ at $562 \mathrm{~nm}$ (curve 1) and $\mathrm{B}_{\mathrm{TE}-\mathrm{TM}}$ at $586 \mathrm{~nm}$. Thus, the sub-bands $\mathrm{B}_{\mathrm{TE}-\mathrm{TM}}$ and $\mathrm{B}_{\mathrm{TM}} \mathrm{TE}$ are located in the same wavelength range, where the light of an arbitrary polarization is reflected. In case of the $\mathrm{B}_{\mathrm{TE}-\mathrm{TE}}$ and $\mathrm{B}_{\mathrm{TM}-\mathrm{TM}}$ sub-bands the reflected light is TE- and TM-polarized, respectively. A qualitative explanation for this spectral splitting into the three sub-bands is given in [19]. Actually, there are three modes interacting with three different refractive indices $-n_{\|},\langle n\rangle$ and $n_{\perp}$, which are responsible for the splitting.

The triple structure of the bands makes quite interesting the question of possible lasing modes depending on a degree of the lying helix deformation. In our particular case the band with $j=3$ in a range $560-620 \mathrm{~nm}$ is of practical interest. That is because this band is well overlapped with the luminescence band of the widely used DCM lasing dye [10].

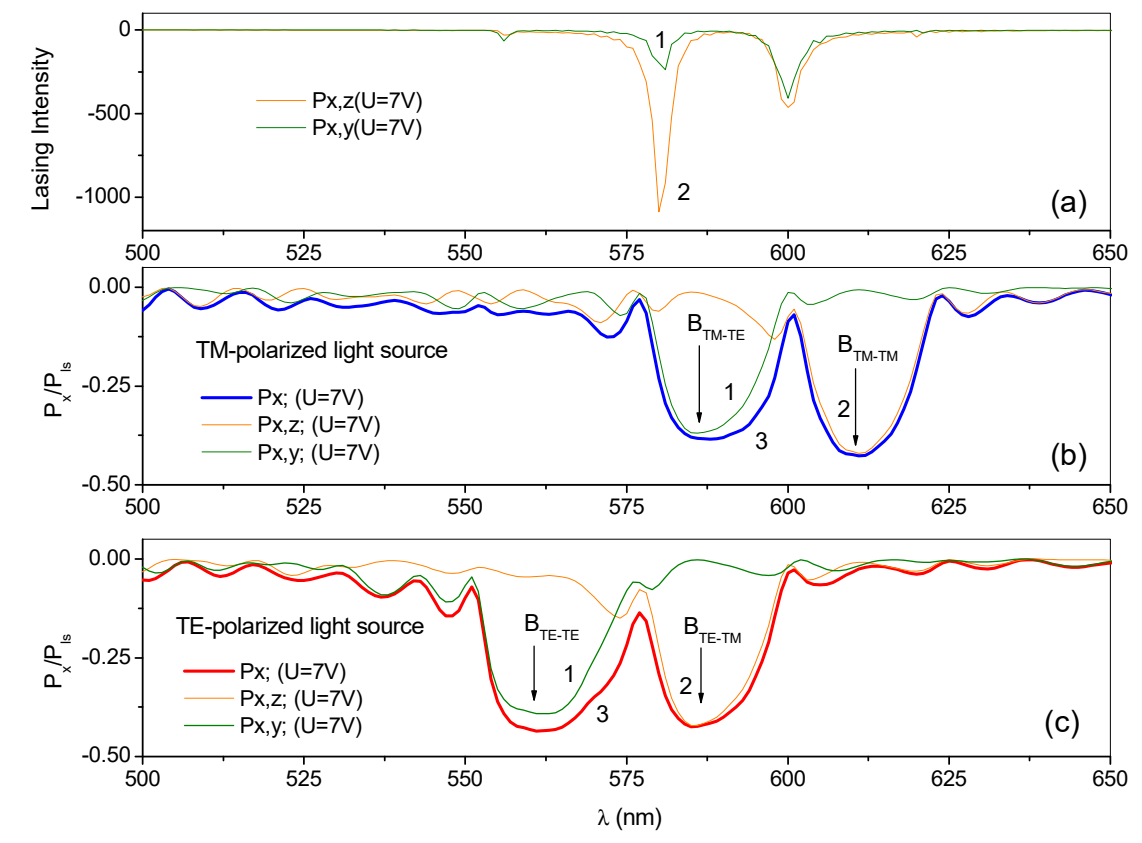

Figure 7. Spectra of the lasing effect (a) and corresponding reflection spectra for the case of a strongly deformed $(\mathrm{U}=7 \mathrm{~V})$ lying helix. The curves 1 and 2 correspond to $\mathrm{y}$ - and z-polarized light of the $x$-component of the Poynting vector $\left(P_{x, y}\right.$ and $\left.P_{x, z}\right)$, respectively. The curves labelled 3 are for the total $P_{x}$ energy flux. The spectra $(\mathbf{b}, \mathbf{c})$ are for the $z-(T M)$ and $y$-polarized (TE polarization) incident light. 
In order to simulate the lasing effect, the CLC medium is virtually made to be amplifying. It is done by using Lorentz oscillators with negative strength. The oscillators' frequencies correspond to a wavelength of $600 \mathrm{~nm}$, while their strengths are set to a value providing a maximum gain coefficient, $g=-k=0.2 \mu \mathrm{m}^{-1}$ at $\lambda=600 \mathrm{~nm}$. In order to make the gain function independent on the light polarization state, the strengths of the two oscillators (along and perpendicular to the LC director) are taken to be equal. Thus, the isotropic gain function $\left(g=g_{\|}=g_{\perp}\right)$ is used, which is favorable for excitation differently polarized lasing modes. It is worth mentioning that in experimental CLCs the gain function can be anisotropic, so not all the simulated modes may be observed in a single experiment. The oscillators damping coefficient $\Gamma_{m}$ is taken to provide the half-height width of the amplifying contour, $\sim 200 \mathrm{~nm}$. The corresponding spectra of the real and imaginary parts of the principal component of the dielectric permittivity tensor used are discussed in the Section 2. Again, the width of the gain spectrum is significantly larger than that, for example, which can be estimated $(\sim 40 \mathrm{~nm})$ from the super-luminescence spectra for an experimental CLC with the DCM lasing dye [16]. However, the used wider gain spectrum allows covering the whole spectral range occupied by the field-induced stop-band in order to simulate all the possible lasing modes, which can be excited in its spectral range.

The simulated lasing intensities correspond to the ratios of the emitted light intensities to the intensities of corresponding spectral components of the impinged light pulse (the excitation scheme is in Figure 3). Because the gain function covers all the stop-band range, the relative intensities of the calculated lasing modes demonstrate the ability of the DHL CLC layer for the resonance amplification of the light inside the whole spectral range of the stop-band. However, in the simulations, the gain function is fixed during evolving the lasing emission, so we are in a frame of the linear optics. In real experiments, for example, at the pulsed optical pumping, the gain function is not fixed, but varies in time during the pumping pulse, and it also depends on the lasing intensity. It means that the real lasing excitation is a nonlinear process, which can result in a difference from the simulated intensities even in a case where the experimental gain function is equivalent to one used in the current simulations.

First, let us look at the case of strongly deformed helix, Figure 7a. In this case the most intensive lasing effect takes place at a wavelength of $580 \mathrm{~nm}$, which corresponds to the short-wavelength edges of the $\mathrm{B}_{\mathrm{TM}} \mathrm{TE}$ and $\mathrm{B}_{\mathrm{TE}-\mathrm{TM}}$ sub-bands. The most significant fraction of the lasing light is TM-polarized (curve 2). The lasing is also excited at the long-wavelength edges $(600 \mathrm{~nm})$ of the same sub-bands $\left(\mathrm{B}_{\mathrm{TM}} \mathrm{TE}\right.$ and $\left.\mathrm{B}_{\mathrm{TE}-\mathrm{TM}}\right)$, but in this case the emitted energy was equally shared between TM- and TE-polarized modes (compare curves 1 and 2). The TE-polarized emission at the short-wavelength edge (556 nm) of the $\mathrm{B}_{\mathrm{TE}-\mathrm{TE}}$ sub-band (curve 1) is only slightly visible, so this lasing mode is less preferable with strong deformation of the DLH.

At the lower deformation of the DLH (Figure 8a) the lasing at $580 \mathrm{~nm}$ disappears. Then the most intensive emission takes place at $612 \mathrm{~nm}$, that corresponds to the long-wavelength edge of $\mathrm{B}_{\mathrm{TM}-\mathrm{TM}}$ sub-band. Slightly less lasing intensity is at $562 \mathrm{~nm}$ that is the edge of the $\mathrm{B}_{\text {TE-TE }}$ sub-band. This edge of the $B_{\text {TE-TE }}$ sub-band is slightly shifted to the longer wavelength $(562 \mathrm{~nm})$ compared to the case of the stronger deformation $(556 \mathrm{~nm})$, which is explained by increasing an effective refractive index for the given mode with decreasing the extent of DLH deformation. While the $\mathrm{B}_{\mathrm{TM}-\mathrm{TM}}$ edge mode shares TMand TE-polarized emissions equally, the $\mathrm{B}_{\mathrm{TE}-\mathrm{TE}}$ mode has higher TE-polarized contribution. As far as $\mathrm{B}_{\mathrm{TM}-\mathrm{TE}}, \mathrm{B}_{\mathrm{TE}-\mathrm{TM}}$ edge lasing, it is slightly pronounced at the long-wavelength edge at $598 \mathrm{~nm}$ only.

Thus, in case of the DLH, depending on extent of the helix deformation, one can get different sets of the lasing modes located at edges of the three sub-bands. At very strong deformation the lasing at edges of the central sub-band is preferable, while decreasing the deformation results in exciting the modes located at the other $\mathrm{B}_{\mathrm{TE}-\mathrm{TE}}$ and $\mathrm{B}_{\mathrm{TM}}$-TM sub-bands. 

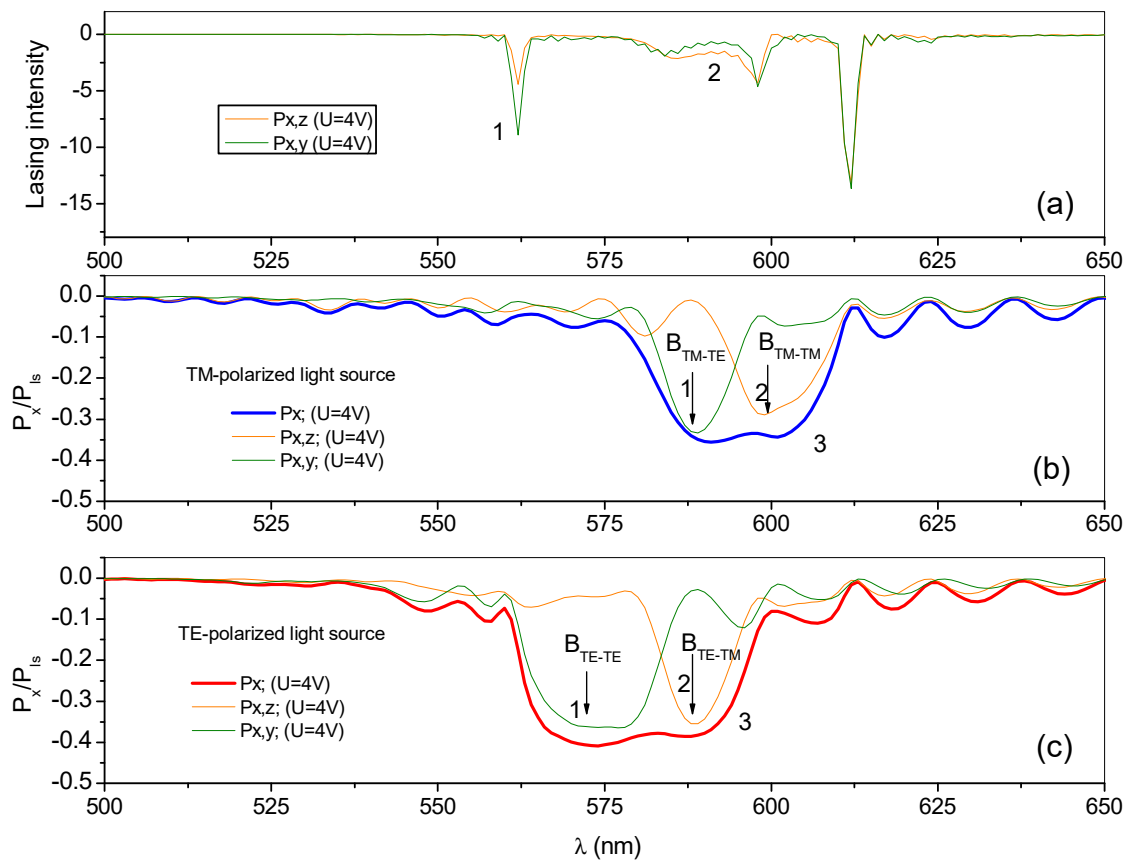

Figure 8. Spectra of the lasing effect (a) and corresponding reflection spectra for the case of a less-deformed $(\mathrm{U}=4 \mathrm{~V})$ lying helix. The curves labelled 1 and 2 correspond to $\mathrm{y}$ - and z-polarized light of the $x$-component of the Poynting vector $\left(P_{x, y}\right.$ and $\left.P_{x, z}\right)$, respectively. The curves labelled 3 are for the total $P_{x}$ energy flux. The spectra $(\mathbf{b}, \mathbf{c})$ are for the $z-(T M)$ and $y$-polarized (TE polarization) incident light.

\section{Conclusions}

In conclusion, the numerical simulations show that the optical properties of the DLH layers are significantly richer compared to ones of the CLC Granjean-plane textures distorted by an electric field perpendicular to the cholesteric helix axis. This difference is caused by appearance of the even harmonics in the spatial distribution of the DLH director field, which leads to additional stop-bands, which are not allowed in case of the field-distorted Granjean-plane textures. All the higher order DLH stop-bands are split into three sub-bands. For each of the sub-bands, the edge-band lasing can be excited depending on extent of deformation of the DLH. Thus, in practice the electric field switching between the lasing modes can be done.

Funding: This work is supported by the Ministry of Science and Higher Education within a state assignment.

Acknowledgments: I am grateful to my colleagues N.M. Shtykov, A.R. Geivandov, B.A. Umanskii and M.V. Gorkunov for many useful discussions regarding the results.

Conflicts of Interest: The author declares no conflict of interest.

\section{References}

1. Oseen, C.W. The theory of liquid crystals. Trans. Faraday Soc. 1933, 29, 883-899. [CrossRef]

2. Il'chishin, I.P.; Tikhonov, E.A.; Shpak, M.T.; Doroshkin, A.A. Stimulated emission lasing by organic dyes in a nematic liquid crystal. JETP Lett. 1976, 24, 303-306.

3. Kopp, V.I.; Zhang, Z.Q.; Genacka, A.Z. Lasing in chiral photonic structures. Prog. Quantum. Electron. 2003, 27, 369-416. [CrossRef]

4. Coles, H.; Morris, S. Liquid-Crystal lasers. Nat. Photonics 2010, 4, 676-685. [CrossRef]

5. Inoue, Y.; Yoshida, H.; Inoue, K.; Fujii, A.; Ozaki, M. Improved lasing threshold of cholesteric liquid crystal lasers with in-plane helix alignment. Appl. Physics Express 2010, 3, 102702. [CrossRef]

6. Blinov, L.M.; Bartolino, R. Liquid Crystal Microlasers; Transworld Research Network: Trivandrum, Kerala, India, 2010. 
7. Palto, S.P.; Shtykov, N.M.; Umanskii, B.A.; Barnik, M.I. Multiwave out-of-normal band-edge lasing in cholesteric liquid crystals. J. Appl. Phys. 2012, 112, 013105. [CrossRef]

8. Belyakov, V.A. From liquid crystals localized modes to localized modes in photonic crystals. J. Lasers Opt. Photonics 2017, 4, 153.

9. Xiang, J.; Varanytsia, A.; Minkowski, F.; Paterson, D.A.; Storey, J.M.D.; Imrie, C.T.; Lavrentovich, O.D.; Palffy-Muhoray, P. Electrically tunable laser based on oblique heliconical cholesteric liquid crystal. Proc. Natl. Acad. Sci. USA 2016, 113, 12925-12928. [CrossRef] [PubMed]

10. Shtykov, N.M.; Palto, S.P.; Umanskii, B.A.; Rybakov, D.O.; Simdyankin, I.V. Fluorescence and lasing in an electric-field-induced periodic structure of a cholesteric liquid crystal. Quantum Electron. 2019, 49, 754-761. [CrossRef]

11. Komitov, L.; Bryan-Brown, G.P.; Wood, E.L.; Smout, A.B.J. Alignment of cholesteric liquid crystals using periodic anchoring. J. Appl. Phys. 1999, 86, 3508-3511. [CrossRef]

12. Wang, C.; Wang, W.; Lin, T. A stable and switchable uniform lying helix structure in cholesteric liquid crystals. Appl. Phys. Lett. 2011, 99, 041108. [CrossRef]

13. Salter, P.S.; Carbone, G.; Jewell, S.A.; Elston, S.J.; Raynes, P. Unwinding of the uniform lying helix structure in cholesteric liquid crystals next to a spatially uniform aligning surface. Phys. Rev. E 2009, 80, 041707. [CrossRef] [PubMed]

14. Ryabchun, A.; Bobrovsky, A. Cholesteric liquid crystal materials for tunable diffractive optics. Adv. Opt. Mater. 2018, 1800335, 1-20. [CrossRef]

15. Nys, I.; Beeckman, J.; Neyts, K. Voltage-controlled formation of short pitch chiral liquid crystal structures based on high resolution surface topography. Opt. Expr. 2019, 2, 11492-11502. [CrossRef] [PubMed]

16. Palto, S.P.; Shtykov, N.M.; Kasyanova, I.V.; Umanskii, B.A.; Geivandov, A.R.; Rybakov, D.O.; Symdyankin, I.V.; Artemov, V.V.; Gorkunov, M.V. Deformed lying helix transition and lasing effect in cholesteric LC layers at spatially periodic boundary conditions. Liq. Cryst. 2019. [CrossRef]

17. Shtrikman, S.; Tur, M. Optical properties of the distorted cholesteric structure. JOSA 1973, 64, 1178-1189. [CrossRef]

18. Belyakov, V.A.; Dmitrienko, V.E. Theory of optical properties of cholesteric liquid crystals in an external field. Sov. Solid State Phys. 1975, 17, 307-309.

19. Palto, S.P.; Barnik, M.I.; Geivandov, A.R.; Kasyanova, I.V.; Palto, V.S. Spectral and polarization structure of field-induced photonic bands in cholesteric liquid crystals. Phys. Rev. E 2015, 92, 032502. [CrossRef] [PubMed]

20. Palto, S.P. Simulation of electrooptical effects and dynamics of ferroelectric liquid crystals. Crystallogr. Rep. 2003, 48, 124-140. [CrossRef]

21. Berreman, D.W. Optics in stratifyed and anisotropic media: $4 \times 4$ matrix formulation. JOSA 1972, 62, 502-510. [CrossRef]

22. Rumpf, R. EMpossible. 2011. Available online: https://empossible.net/academics/emp5304/ (accessed on 5 September 2019).

23. De Gennes, P.G.; Prost, J. The Physics of Liquid Crystals; Oxford University Press: New York, NY, USA, 1993.

(C) 2019 by the author. Licensee MDPI, Basel, Switzerland. This article is an open access article distributed under the terms and conditions of the Creative Commons Attribution (CC BY) license (http://creativecommons.org/licenses/by/4.0/). 